\title{
Latitudinal distribution of the solar wind properties in the low- and high-pressure regimes: Wind observations
}

\author{
C. Lacombe ${ }^{1}$, C. Salem ${ }^{1}$, A. Mangeney ${ }^{1}$, J.-L. Steinberg ${ }^{1}$, M. Maksimovic ${ }^{2,1}$, J. M. Bosqued ${ }^{3}$ \\ ${ }^{1}$ DESPA/CNRS, Observatoire de Paris, 92195 Meudon, France \\ 2 Space Science Dept. of ESA, ESTEC, 2200 AG Noordwijk, The Netherlands \\ ${ }^{3}$ CESR/CNRS, Université Paul Sabatier, BP 4346, 31029 Toulouse, France
}

Received: 29 November 1999 / Revised: 27 March 2000 / Accepted: 6 April 2000

\begin{abstract}
The solar wind properties depend on $\lambda$, the heliomagnetic latitude with respect to the heliospheric current sheet (HCS), more than on the heliographic latitude. We analyse the wind properties observed by Wind at 1 AU during about 2.5 solar rotations in 1995, a period close to the last minimum of solar activity. To determine $\lambda$, we use a model of the HCS which we fit to the magnetic sector boundary crossings observed by Wind. We find that the solar wind properties mainly depend on the modulus $|\lambda|$. But they also depend on a local parameter, the total pressure (magnetic pressure plus electron and proton thermal pressure). Furthermore, whatever the total pressure, we observe that the plasma properties also depend on the time: the latitudinal gradients of the wind speed and of the proton temperature are not the same before and after the closest HCS crossing. This is a consequence of the dynamical stream interactions. In the low pressure wind, at low $|\lambda|$, we find a clear maximum of the density, a clear minimum of the wind speed and of the proton temperature, a weak minimum of the average magnetic field strength, a weak maximum of the average thermal pressure, and a weak maximum of the average $\beta$ factor. This overdense sheet is embedded in a density halo. The latitudinal thickness is about $5^{\circ}$ for the overdense sheet, and $20^{\circ}$ for the density halo. The HCS is thus wrapped in an overdense sheet surrounded by a halo, even in the noncompressed solar wind. In the high-pressure wind, the plasma properties are less well ordered as functions of the latitude than in the low-pressure wind; the minimum of the average speed is seen before the HCS crossing. The latitudinal thickness of the high-pressure region is about $20^{\circ}$. Our observations are qualitatively consistent with the numerical model of Pizzo for the deformation of the heliospheric current sheet and plasma sheet.
\end{abstract}

Key words: Interplanetary physics (solar wind plasma)

Correspondence to: C. Lacombe

e-mail: catherine.lacombe@obspm.fr

\section{Introduction}

Observations of the latitudinal distribution of the solar wind properties (velocity, density, temperatures) are necessary to test models of the solar wind, and models of the heliospheric plasma sheet (HPS) which surrounds the heliospheric current sheet (HCS). The exploration by Ulysses of the heliographic latitude range $-80^{\circ}(\mathrm{S}) \leq \lambda_{G} \leq 80^{\circ}(\mathrm{N})$ yielded the density and the speed of the wind as functions of $\lambda_{G}$ (Phillips et al., 1995; Issautier et al., 1997). Latitudinal models of the solar wind have been compared with the Ulysses data averaged over the 26 days of a solar rotation (Lima and Tsinganos, 1996; Suess et al., 1996). In these models the HCS, i.e. the magnetic equator, is assumed to be in the plane of the heliographic equator: the axis of the dipolar solar magnetic field is along the solar rotation axis. These axisymmetric models neglect the dynamical interactions between the fast and the slow streams of the wind. They also assume north-south symmetry. These models can be fitted to the Ulysses data, in which the density $N_{p}$ is about 2.7 times larger and the speed $V_{s w}$ 1.8 times smaller at $\lambda_{G}=0^{\circ}$ than at $\left|\lambda_{G}\right|=80^{\circ} . N_{p}$ and $V_{s w}$ averaged over a solar rotation may thus be considered as controlled by $\lambda_{G}$. On the other hand, the high-resolution measurements of $N_{p}$ and $V_{s w}$ display several peaks and troughs for $-40^{\circ} \leq \lambda_{G} \leq 20^{\circ}$ (Phillips et al., 1995). The occurrence of these peaks and troughs is not related to $\lambda_{G}$. Thus, the non-averaged density and speed are not controlled by $\lambda_{G}$.

As shown for instance by Zhao and Hundhausen (1981), the solar wind properties at $1 \mathrm{AU}$ are more closely dependent on the heliomagnetic latitude $\lambda$ than on the heliographic latitude $\lambda_{G}$. These authors assumed that the HCS was a plane tilted at about $30^{\circ}$ to the solar heliographic equator; $\lambda$ is the latitude with respect to this planar HCS, counted in an heliographic meridian plane. However, the HCS is generally not a plane. Bruno et al. (1986) avoided this hypothesis: they derived the shape of the warped and tilted HCS close to the Sun 
from the maximum brightness curve at 1.75 solar radii observed in the solar corona by the HAO K-coronameter. They did not determine the latitude $\lambda$ of the spacecraft with respect to the HCS but its angular distance $\delta$ from the HCS, in a direction perpendicular to it. Bruno et al. (1986) only considered solar wind regions without stream interactions. In 1976 and 1977, close to a minimum of solar activity, they found a clear dependence of $V_{s w}, N_{p}$ and the proton temperature $T_{p}$ on $\delta$ : $V_{s w}$ and $T_{p}$ were minimum, and $N_{p}$ was maximum at low $\delta$. The latitudinal profiles of $N_{p}$ and $T_{p}$ were symmetrical about the HCS; $V_{s w}$ was less symmetrical.

The heliomagnetic latitude $\lambda$ (or $\delta$ ) is not a local quantity which could be measured by a spacecraft; it can only be estimated using models of the HCS at $1 \mathrm{AU}$, relying on different observations in the photosphere and in the corona (see the comparative analysis of these models made by Neugebauer et al., 1998). The HCS model is always assumed to be a warped but continuous surface all around the Sun, from a few solar radii to several AU. In the present work, we shall start from the classic model of the HCS (Hoeksema et al., 1983) described in Sect. 2, but we shall fit it to the sector boundary crossings observed on Wind over 70 days in 1995. With this fitted HCS, we obtain the fitted heliomagnetic latitude $\lambda_{f}$ of Wind, which varies between $-20^{\circ}$ and $30^{\circ}$ during the interval considered. This relatively large interval of $\lambda_{f}$ justifies the use of the data of Wind for a study of latitudinal effects in the solar wind.

We find that $N_{p}, V_{s w}$ and $T_{p}$ observed at $1 \mathrm{AU}$ on Wind are indeed functions of the heliomagnetic latitude $\lambda_{f}$, but these functions are different in two kinds of wind: the low-pressure wind, where the total pressure (magnetic pressure plus electron and proton thermal pressure) is below about $5 \times 10^{-11} \mathrm{~Pa}$, and the highpressure wind. A clear minimum of $V_{s w}$ and $T_{p}$ at low latitudes is seen only in the low-pressure wind. In both kinds of wind, we find that the profiles of $N_{p}, V_{s w}$ and $T_{p}$ display a crude north-south symmetry. But we show in Sect. 5 that the latitudinal gradients of $V_{s w}$ and $T_{p}$ are not the same before and after an HCS crossing: even in the low-pressure wind there are indications of the part played by the fast stream-slow stream interactions. We show that our observations are qualitatively consistent with the MHD model of Pizzo (1994). In this model the stream interactions are taken into account, and the deformations of the HCS and of the HPS are calculated (at 5 AU).

We shall particularly address the question of the latitudinal distribution of the density in the solar wind. Borrini et al. (1981) (see also Gosling et al., 1981) analysed several tens of magnetic sector boundaries or HCS crossings. They found two types of overdense regions in the ecliptic plane: (1) the stream-free overdensities which are found close to the HCS even if the HCS crossing is not followed by a fast stream within $24 \mathrm{~h}$; (2) overdensities observed just before fast streams, and due to dynamical interactions in the wind. These two kinds of overdensities are often superimposed close to the HCS. We shall consider all the solar wind regions in the ecliptic plane where $N_{p}$ measured by Wind is significantly enhanced over a time period devoid of magnetic clouds or strong interplanetary shocks. Over this continuous interval of 70 days we can test whether there is always an overdensity at the sector boundary. The existence and the strength of this overdensity are important parameters for studies of the propagation of low-frequency radio waves in the interplanetary medium (Steinberg et al., 2000).

\section{The data}

The proton density $N_{p}$ on Wind is measured by the SWE experiment (Ogilvie et al., 1995), the magnetic field by the MFI experiment (Lepping et al., 1995), and the temperatures $T_{e}$ and $T_{p}$ by the 3D-plasma experiment (Lin et al., 1995). We used the key parameters (KP) data of these three experiments: they have a time resolution of $\simeq 1.5 \mathrm{~min}$ (930 points per day). We also used the detailed electron distribution functions measured by the 3D-plasma experiment up to about $1 \mathrm{keV}$. We integrated these electron distribution functions to calculate the electron heat flux vector every $1.5 \mathrm{~min}$. All the KP data and the calculated quantities have been smoothed and resampled to give only 125 points per day (averages over about $10 \mathrm{~min}$ ).

The interval chosen is close to the solar cycle minimum. It extends from May 15, 1995, to July 23, 1995 , i.e. from 135.0 to 205.0 decimal days of the year. It was selected because no transient, magnetic cloud or strong interplanetary shock was observed over the 70 days (Sanderson et al., 1998). Until the end of June, Wind was around the Lagrange point, more than 200 Earth radii $\left(R_{E}\right)$ away from the Earth; in July, Wind went back towards the Earth, and it was at about $100 R_{E}$ away from it on July 24. From day 157.6 to 161.6, there are frequent and large data gaps in the electron distribution functions, so that the calculated averages of the electron heat flux can be biased. From day 185.1 to 187.6 , neither the distribution functions nor the KP data were completely corrected for the effect of the photoelectrons. During the rest of the interval (days 135.0 to 185.1 , days 187.6 to 205.0 ), the perturbations to the distribution functions due to the photoelectrons can be neglected in the calculation of the heat flux.

The basis of our determination of the heliomagnetic latitude of Wind at $1 \mathrm{AU}$ is the classic model of Hoeksema et al. (1983) for the HCS, which is available on line and used in several studies (Sanderson et al., 1998; Ma et al., 1999). In this model, the position of the neutral line of the photospheric magnetic fields is deduced from the observations of the Wilcox Solar Observatory. The resolution of the synoptic charts of the photospheric field is $5^{\circ}$ in latitude and in longitude. Above the photosphere, the magnetic field is assumed to be potential and is calculated up to 2.5 solar radii. Higher up the magnetic field is assumed to be radial, so that the heliographic latitude $\lambda_{h c s}$ of the neutral line (the HCS) at $1 \mathrm{AU}$ is the same as at 2.5 solar radii, with a time delay of 5 days. The delay of 5 days, corresponding 
to a solar wind speed of $\simeq 350 \mathrm{~km} / \mathrm{s}$, results from a statistical analysis made by Hoeksema et al. (1982); it is not exactly valid for each HCS crossing. We shall thus modify the classic model to take into account the crossings of the HCS observed at $1 \mathrm{AU}$ : they sometimes occur one day (or more) before or after the predicted crossing.

\section{Determination of the heliomagnetic latitude of Wind}

The heliomagnetic latitude $\lambda_{H}$ of the Earth (i.e. of Wind) with respect to the HCS, on a day $d$, can be calculated with the heliographic latitude of the HCS $\lambda_{\text {hcs }}$ estimated on the day $d-5$ by the method of Hoeksema et al. (1982, 1983), and with the heliographic latitude of the Earth $\lambda_{E}$ :

$\lambda_{H}(d)=\lambda_{E}(d)-\lambda_{h c s}(d-5)$

The thin line in Fig. 1a is a plot of $\lambda_{H}$ as a function of time in days. Eleven HCS crossings $\left(\lambda_{H}=0\right)$ are predicted during this interval. Figure $1 \mathrm{~b}$ displays $a z_{B}$, the azimuth of the solar wind magnetic field $\mathbf{B}$ with respect to the Sun direction, measured on Wind and averaged over $2.4 \mathrm{~h}$ (10 points per day). There is a good overall agreement between the $\lambda_{H}$ prediction and the measured $a z_{B}$ : the azimuth is generally close to $135^{\circ}$ (antisunward along the Parker spiral) when $\lambda_{H}>0$, and to $-45^{\circ}$ (sunward along the spiral) when $\lambda_{H}<0$. The dashed line at $a z_{B}=45^{\circ}$ is the boundary between the sunward and the antisunward magnetic sector.

A jump of $a z_{B}$ will be considered as a current sheet (CS) crossing when it separates regions of well-defined north and south polarity of $\mathbf{B}$, with $\mathbf{B}$ more or less along the Parker spiral $a z_{B} \simeq-45^{\circ}$ or $135^{\circ}$. These CS crossings are shown by diamonds in Fig. $1 \mathrm{~b}$. In spite of the overall agreement between $\lambda_{H}$ and $a z_{B}$, there are delays between some of the predicted HCS crossings $\left(\lambda_{H}=0\right)$ and the CS crossings: for instance $\lambda_{H}=0$ on the days 156 and 162, while Wind actually crosses current sheets on the days $158-159$ and 165 . On the other hand, there are many more observed CS crossings than predicted HCS crossings. We have thus to verify whether all the
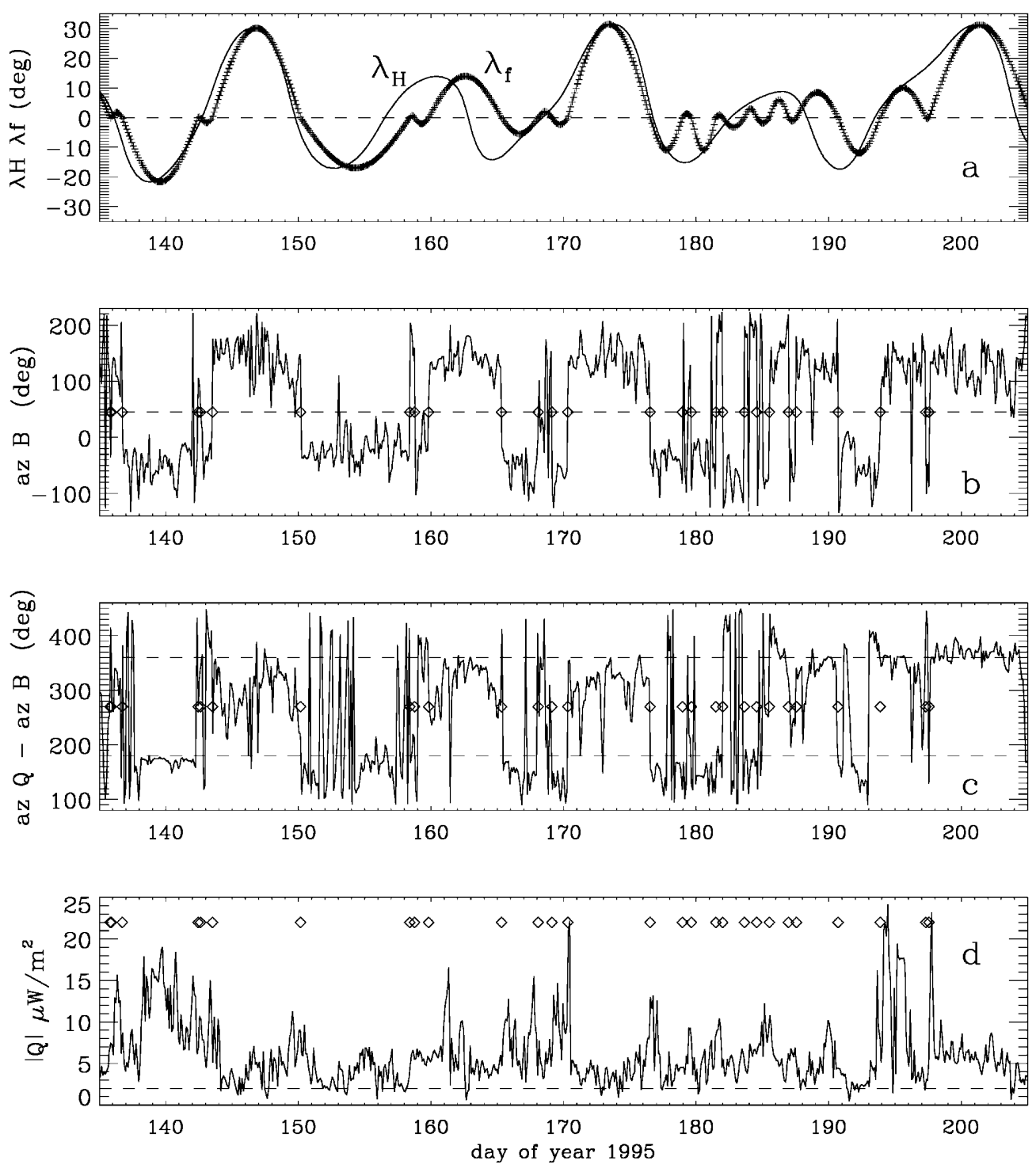

Fig. 1a-d. Seventy consecutive days of 1995 (May 15 to July 23). a The latitude of Wind with respect to the magnetic sector boundary or HCS. Thin line: $\lambda_{H}$ estimated by Hoeksema. Thick line: $\lambda_{f}$ adjusted with the HCS crossings observed on Wind (Sect. 3). b The magnetic field azimuth averaged over $2.4 \mathrm{~h}$. The diamonds indicate the time of the current sheet crossings. c The difference between the azimuth of the heat flux and the azimuth of the magnetic field, when $|Q|$ is larger than $2 \mu \mathrm{W} / \mathrm{m}^{2}$. d The modulus $|Q|$ of the total electron heat flux, averaged over $2.4 \mathrm{~h}$ 
observed CS crossings can be considered as crossings of the HCS.

The electron heat flux data can help to make this verification (Szabo et al., 1999). The modulus $|Q|$ of the heat flux vector averaged over $2.4 \mathrm{~h}$ is shown in Fig. 1d during the 70 days considered. $\mathbf{Q}$ is the total electron heat flux up to about $1 \mathrm{keV}$. Earlier studies showed that the electron heat flux is generally antisunward, along the local B (Feldman et al., 1975; Scime et al., 1994). Figure $1 \mathrm{c}$ is a plot of the difference between the azimuth of $\mathbf{Q}$ and the azimuth of $\mathbf{B}, a z_{Q}-a z_{B}$, for the time intervals when $|Q| \geq 2 \mu \mathrm{W} / \mathrm{m}^{2}$ : we have not taken into account the intervals when $|Q|$ is weaker than $2 \mu \mathrm{W} / \mathrm{m}^{2}$, when the direction of $\mathrm{Q}$ is poorly determined. In Fig. 1c, $a z_{Q}-a z_{B}$ is generally equal to $180^{\circ}$ when $\mathbf{B}$ is sunward along the spiral $\left(a z_{B} \simeq-45^{\circ}\right)$, and generally equal to $360^{\circ}$ when $\mathbf{B}$ is antisunward along the spiral $\left(a z_{B} \simeq 135^{\circ}\right)$. Thus the observed total electron heat flux is generally antisunward.

In a simple ideal HCS crossing the sign of $a z_{B}$ changes while $\mathbf{Q}$ remains antisunward so that, if $a z_{Q}-a z_{B}$ is counted between $90^{\circ}$ and $450^{\circ}, a z_{B}$ and $a z_{Q}-a z_{B}$ jump in the same direction: this occurs on days 136.7, 150.2, 165.3, 170.3, 176.5, 187.0, 187.6 and 190.7 (Fig. 1b, c). On these days, the CS crossings indicated by diamonds can thus be considered as crossings of the HCS. For the other crossings in Fig. $1 \mathrm{~b}, a z_{B}$ and $a z_{Q}-a z_{B}$ are anticorrelated: see e.g. the interval 158 to 160 . This implies that the heat flux is sunward. According to Kahler et al. (1998) and Crooker (1999), a sunward heat flux can be found when the field lines are folded or coiled.

Our aim, however, is not to analyse the small-scale structures close to the current sheets but to determine the latitude of Wind with respect to the sector boundary. In spite of small intervals of sunward heat flux, all the CS crossings in Fig. $1 \mathrm{~b}$ can be considered as close to crossings of the sector boundary or of the HCS because they occur between two regions where $\mathbf{Q}$ is antisunward and where the $\mathbf{B}$ polarities in the spiral direction are opposite. We have only rejected a couple of $\mathbf{B}$ field reversals which occurred on day 153 , with a sunward heat flux between the two reversals: these current sheet crossings are probably not crossings of the HCS because they occurred in the middle of a high-speed flow (Fig. 2b) where $V_{s w}$ was larger than $570 \mathrm{~km} / \mathrm{s}$. Figure 2b, c displays $V_{s w}$ and $N_{p}$; Fig. 2a, d will be discussed in Sects. 4 and 5.

After having selected the HCS crossings among the observed set of CS crossings, we adjust the $\lambda_{H}$ latitude estimated by the classic model to the HCS crossings. To do that, we assume that the fitted latitude $\lambda_{f}$ is equal to $0^{\circ}$ at all the observed HCS crossings, and that the variation of $\lambda_{f}$ is made of sinusoidal arcs with the same amplitude and sign as the closest predicted $\lambda_{H}$ arc. When the interval between two HCS crossings is small (for instance days $158-160$ or days $168-171$ ) the amplitude of the arc is assumed to be small and proportionnal to the time interval between the two crossings. The resulting fitted $\lambda_{f}$ curve is the thick line in Fig. 1a.

The small waves which appear on the fitted $\lambda_{f}$ curve do not imply that the HCS is so wavy perpendicular to the ecliptic plane. Multiple current sheets can be present in the streamer belt, as in the model of Crooker et al. (1993), while our model relies on the assumption that the HCS is a continuous sheet in the streamer belt. Near these multiple current sheets, the latitude with respect to the sector boundary is low, even if it is not exactly zero. On the other hand, when $V_{s w}$ is large over several days, the latitude with respect to the HCS is certainly large, as in Hoeksema's calculations. Our $\lambda_{f}$ curve (Fig. 1a) is simply a continuous sinusoidal interpolation between the points where $\lambda_{f}$ is certainly large, and the CS crossings where $\lambda_{f}$ is certainly low, with the sign of $\lambda_{f}$ adjusted to the sign of $a z_{B}$. Note that there are CS crossings not predicted by the classic model, for instance on days 170 and 197. However on these two days, these are inflexions of the $\lambda_{H}$ curve for the coronal fields, corresponding to the changes of $a z_{B}$ observed at $1 \mathrm{AU}$.

In what follows, we shall consider that the fitted $\lambda_{f}(t)$ curve is a good estimation of the latitude of Wind with respect to the heliospheric current sheet, as a function of time, although we made several assumptions in deriving it: continuous heliospheric current sheet, arbitrary sinusoidal shape, more or less arbitrary amplitudes, uncertain identification of the position of the heliospheric current sheet crossings. As the resolution of the synoptic charts of the photospheric magnetic field is $5^{\circ}$, the uncertainty on $\lambda_{H}$ and on $\lambda_{f}$ is at least $5^{\circ}$.

\section{Plasma properties as functions of the heliomagnetic latitude}

Figure $3 \mathrm{a}$ is a plot of the proton density $(10 \mathrm{~min}$ averages), as a function of the latitude $\lambda_{H}$. The average density peaks in the range $-20^{\circ}<\lambda_{H}<20^{\circ}$. There are minimum values of $N_{p}$ weaker than $5 \mathrm{~cm}^{-3}$ at any $\lambda_{H}$. Figure $3 \mathrm{~b}$ is a plot of $N_{p}$ as a function of $\lambda_{f}$. That density distribution is narrower than that in Fig. 3a: this better organization of $N_{p}$ can be an a posteriori indication that our fitted latitude is valid. The densities larger than $10 \mathrm{~cm}^{-3}$ observed at $\lambda_{f} \simeq 20^{\circ}$ are due to a weak forward interplanetary shock which occurred at 05:30 UT on July 22 (day 203.23); four even weaker reverse interplanetary shocks were observed on days $144.19,150.77$, 170.57, and 177.15 (crosses in Fig. 2b).

The fact that $N_{p}$ is generally larger than 5 to $7 \mathrm{~cm}^{-3}$ at $\left|\lambda_{f}\right| \leq 4^{\circ}$ implies that overdense regions are always observed close to the HCS or close to current sheets (see McComas et al., 1989; Crooker et al., 1996). The overdensity at low $\left|\lambda_{f}\right|$ seems to consist of a continuous sheet with a density at least twice larger than at high $\left|\lambda_{f}\right|$. At high positive $\lambda_{f}, N_{p}$ is weaker than 3 to $5 \mathrm{~cm}^{-3}$, consistent with the values observed by Ulysses at high heliographic latitudes.

Figure 4a allows a comparison of the latitudinal variation of $N_{p}$ found by Bruno et al. (1986) with that found on Wind. The solid line is a plot of the average value of our sample as a function of $\lambda_{f}$ in bins of $2.5^{\circ}$, the dashed lines are the average plus or minus the standard deviation in each bin for the 10 min averages. The two series of squares indicate the densities found by 

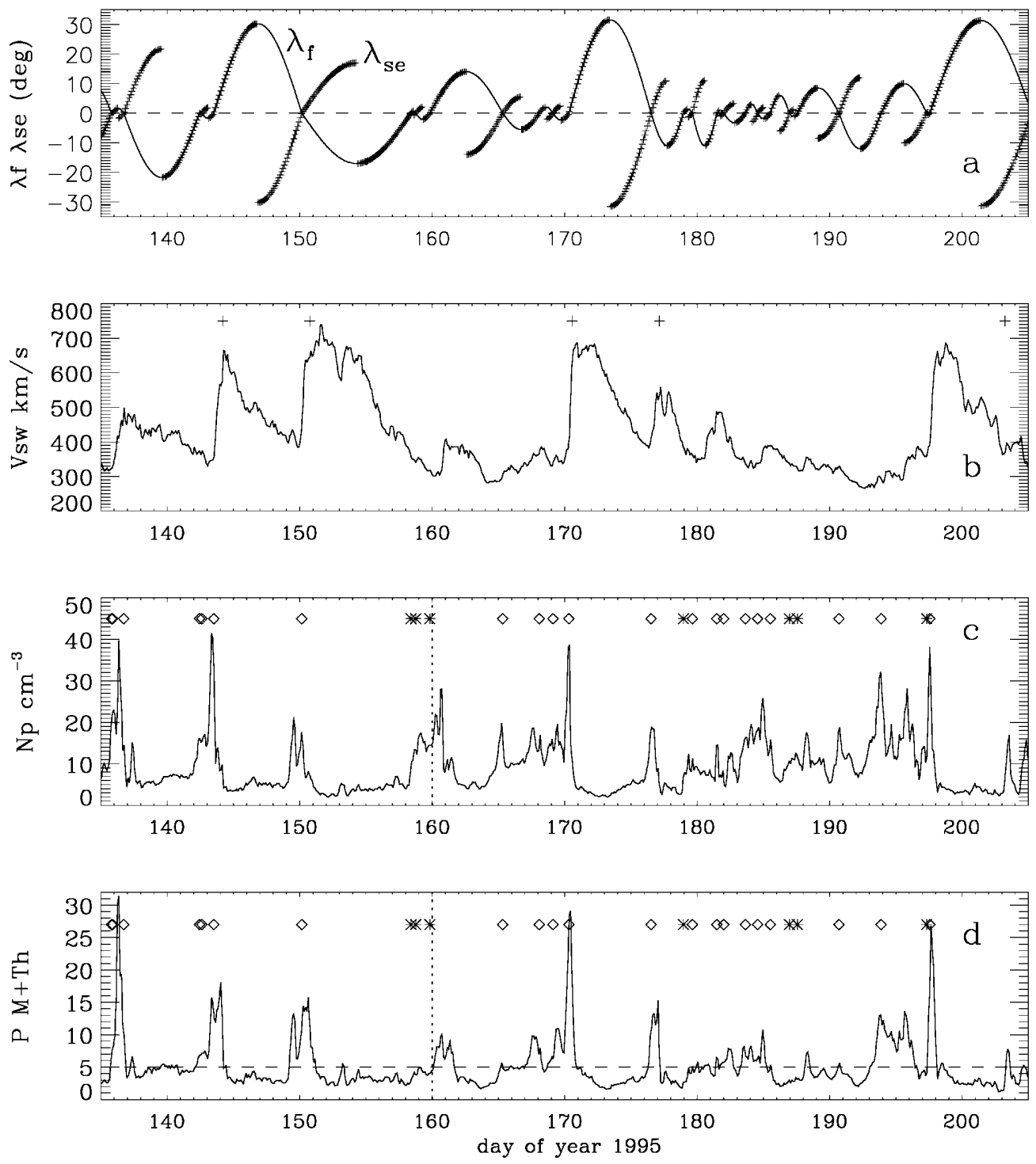

Fig. 2. a The latitude of Wind with respect to the magnetic sector boundary or HCS. Thin line: $\lambda_{f}$. Thick line: $\lambda_{\text {se }}$, the superposed epoch latitude defined in Sect. 5. b The wind speed averaged over $2.4 \mathrm{~h}$. The crosses indicate the time of interplanetary shocks. c The proton density averaged over $2.4 \mathrm{~h}$. The diamonds indicate the time of the current sheet crossings in a high-pressure wind, the stars in a low-pressure wind. d The magnetic plus thermal pressure (Eq. 2), units $10^{-11} \mathrm{~Pa}$. The dashed vertical line at the beginning of the day 160 is the boundary between non-compressive and compressive density enhancements
Bruno et al. (1986) and scaled to $1 \mathrm{AU}$, as functions of the distance $\delta$ from the HCS of their sample, respectively in 1976 and 1977, close to the minimum of the solar cycle. Our average density is consistent with the average densities observed by Bruno et al. (1986).

In their work, Borrini et al. (1981) made a superposed epoch analysis of sector boundaries. They selected welldefined sector boundaries or HCS crossings. A density peak was observed in the stream-free HCS crossings, and a stronger peak in the stream-associated HCS crossings. In the present work, we wish to see whether overdensities are observed through any stream-free sector boundary, even if the sector boundary is not so well defined.

The overdensities close to the HCS and the overdensities close to the fast wind boundary are often but not always superimposed. We need a local criterion to distinguish the overdensities close to the HCS, which probably form a sheet as continuous as the HCS itself, from the overdensities close to the fast wind boundary, which are probably not continuous but localised in longitude, at the corotating interaction regions. The magnetic pressure plus the thermal pressure
$P=P_{T h}+P_{M}=N_{p} k_{B}\left(T_{e}+T_{p}\right)+B^{2} / 2 \mu_{0}$

can be a good criterion to distinguish the two types of overdensities. Indeed, according to Gosling et al. (1977, 1981) density enhancements without enhancements of the pressure $P$ are currently observed in the solar wind (non compressive density enhancements, NCDE); conversely, the pressure $P$ peaks inside the overdensities related to the compression by the fast streams. Figure 2b-d gives $V_{s w}, N_{p}$ and $P$ as functions of time for the considered interval. (The temperatures are not shown; $T_{e}$ varies between 8 and $30 \mathrm{eV}$, and $T_{p}$ between 3 and $85 \mathrm{eV})$. The vertical dotted line in Fig. 2c, d, at the beginning of day 160, indicates the end of a NCDE and the beginning of a compressed interval. $P$ is weak (2 to $5 \times 10^{-11} \mathrm{~Pa}$ ) while $N_{p}$ is large (NCDE) on days 158 and 159 where sector boundary crossings occur. But $P$ peaks to $10 \times 10^{-11} \mathrm{~Pa}$ on day 160 , in the overdensity observed just before the increase of the wind speed. The stars in Fig. 2c, d indicate the HCS crossings which occur in the low-pressure wind $P \leq 5 \times 10^{-11} \mathrm{~Pa}$, the diamonds are the crossings for $P>\overline{5} \times 10^{-11} \mathrm{~Pa}$. 

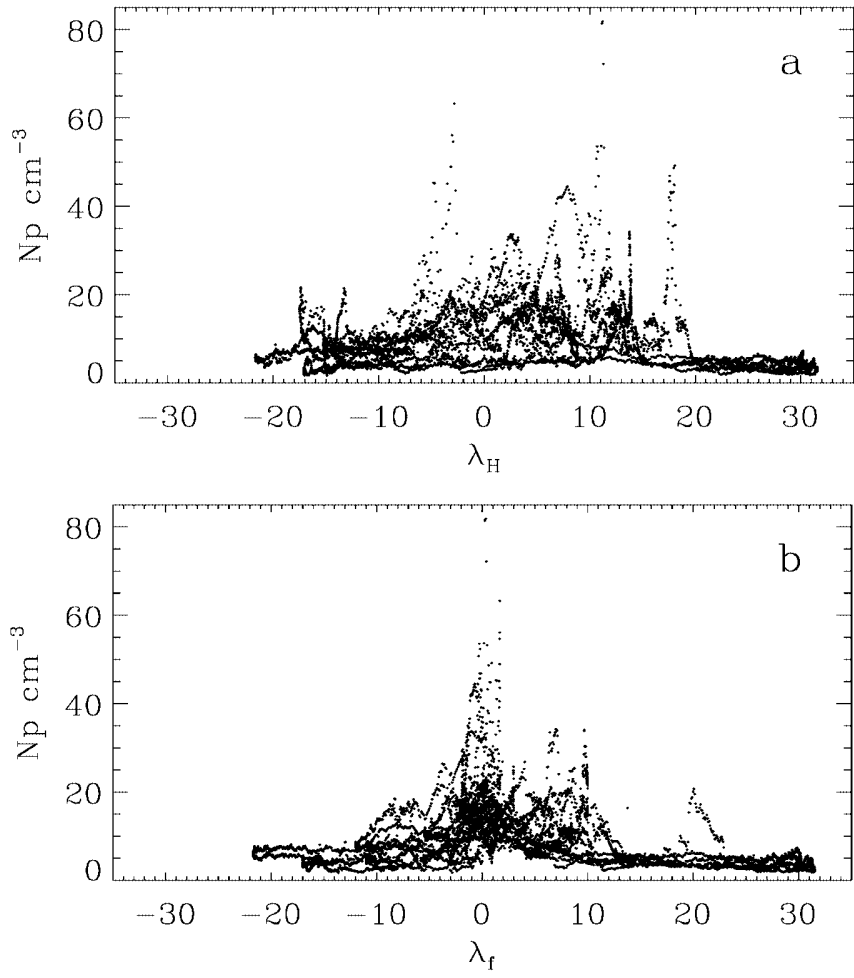

Fig. 3a, b. Seventy consecutive days. a Scatter plot of the proton density (10 min averages) as a function of $\lambda_{H}$ (Eq. 1), the latitude of Wind with respect to the HCS predicted by Hoeksema. b Same scatter plot, as a function of the fitted heliomagnetic latitude $\lambda_{f}$ defined in Sect. 3

Figure $4 \mathrm{~b}$ displays the average density observed during the whole interval as a function of the fitted latitude $\lambda_{f}$. The thin line is $N_{p}$ observed whatever the pressure $P$ may be, already shown in Fig. 4a. The thick line is the average observed when $P$ is smaller than $5 \times 10^{-11} \mathrm{~Pa}$, in the low-pressure wind, outside the interaction regions. We conclude that there is a continuous density sheet in the non-compressed solar wind, with an average density larger than $10 \mathrm{~cm}^{-3}$ and a latitudinal thickness of about $5^{\circ}$.

$N_{p}, V_{s w}$ and $T_{p}$ as functions of $\lambda_{f}$ are shown in the left panels of Fig. 5 for the low-pressure solar wind $\left(P \leq 5 \times 10^{-11} \mathrm{~Pa}\right)$, and in the right panels for the high-pressure wind $\left(P>5 \times 10^{-11} \mathrm{~Pa}\right)$. The similarity of the Figures for $V_{s w}$ and $T_{p}$ illustrates the well-known correlation between $T_{p}$ and $V_{s w}$ (Hundhausen et al., 1970). We note that the distributions of $N_{p}, V_{s w}$ and $T_{p}$ are nearly the same at south and north heliomagnetic latitudes: they mainly depend on $\left|\lambda_{f}\right|$. We also note that speeds as large as $700 \mathrm{~km} / \mathrm{s}$ are found in the highpressure wind at low $\left|\lambda_{f}\right|$. This implies that high speeds are not only found in the dilute low-pressure plasma sampled by Ulysses at heliographic latitudes $\left|\lambda_{G}\right|>40^{\circ}$.

\section{Heliomagnetic latitude for superposed epochs}

Bruno et al. (1986) found that the profiles of the solar wind properties as functions of the angular distance from the HCS were generally symmetrical with respect to the HCS crossing at $\lambda=0$ : these properties mainly
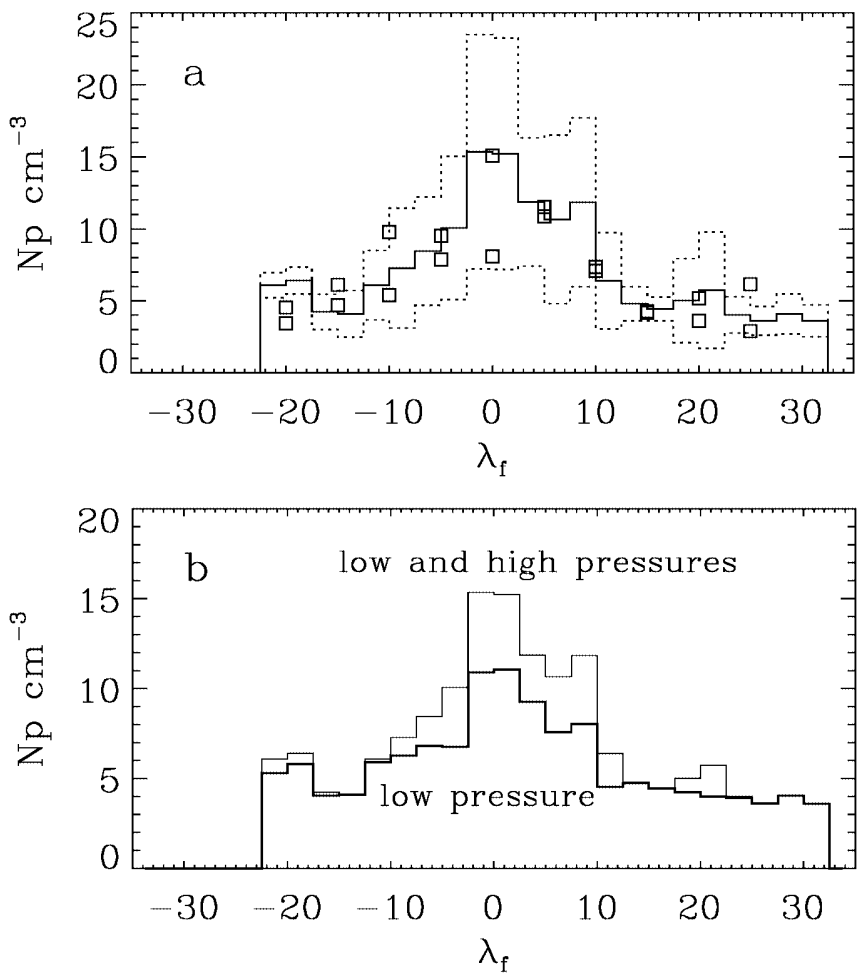

Fig. 4. a The thick line and the dashed lines are respectively plots of the average density over 70 days, and the same density plus or minus the standard deviation (for data averaged over $10 \mathrm{~min}$ ). The two series of squares indicate the values found by Bruno et al. (1986) as functions of the angular distance from the HCS in 1976 and in 1977. b The thick line gives the average density as a function of $\lambda_{f}$ in the lowpressure solar wind, that is when the total pressure (Eq. 2) is smaller than $5 \times 10^{-11} \mathrm{~Pa}$. The thin line is recalled for comparison: it is the average density whatever the pressure shown in the upper panel

depend on $|\lambda|$. With a superposed epoch analysis, Borrini et al. (1981) found that the profiles of the same properties as functions of the time $t$ were not symmetrical with respect to the HCS crossing at $t=0$, at least during stream-associated crossings: the minima of $V_{s w}$ and $T_{p}$ are observed before the HCS crossing. An asymmetry of $N_{p}$ and of the level of the density fluctuations was also observed by Huddleston et al. (1995). We wish to test whether the density gradient as a function of $|\lambda|$ is steeper before or after the HCS crossing; we thus combine an analysis in terms of the fitted heliomagnetic latitude $\lambda_{f}$ and a superposed epoch analysis by defining a superposed epoch latitude $\lambda_{\text {se }}$ which has the same modulus $\left|\lambda_{s e}\right|=\left|\lambda_{f}\right|$, but is taken as negative before the closest HCS crossing and positive after. $\lambda_{s e}$ is drawn as a thick line in Fig. 2a, the thin line is $\lambda_{f}$.

\subsection{Low-pressure wind}

The left panels of Fig. 6 are scatter plots of five solar wind parameters as functions of $\lambda_{\text {se }}$ in the low-pressure wind. The right panels give plots of the average values of these parameters. The thick solid line is the average value, and the dotted lines the average plus or minus the standard deviation for the $10 \mathrm{~min}$ data. The thin solid 

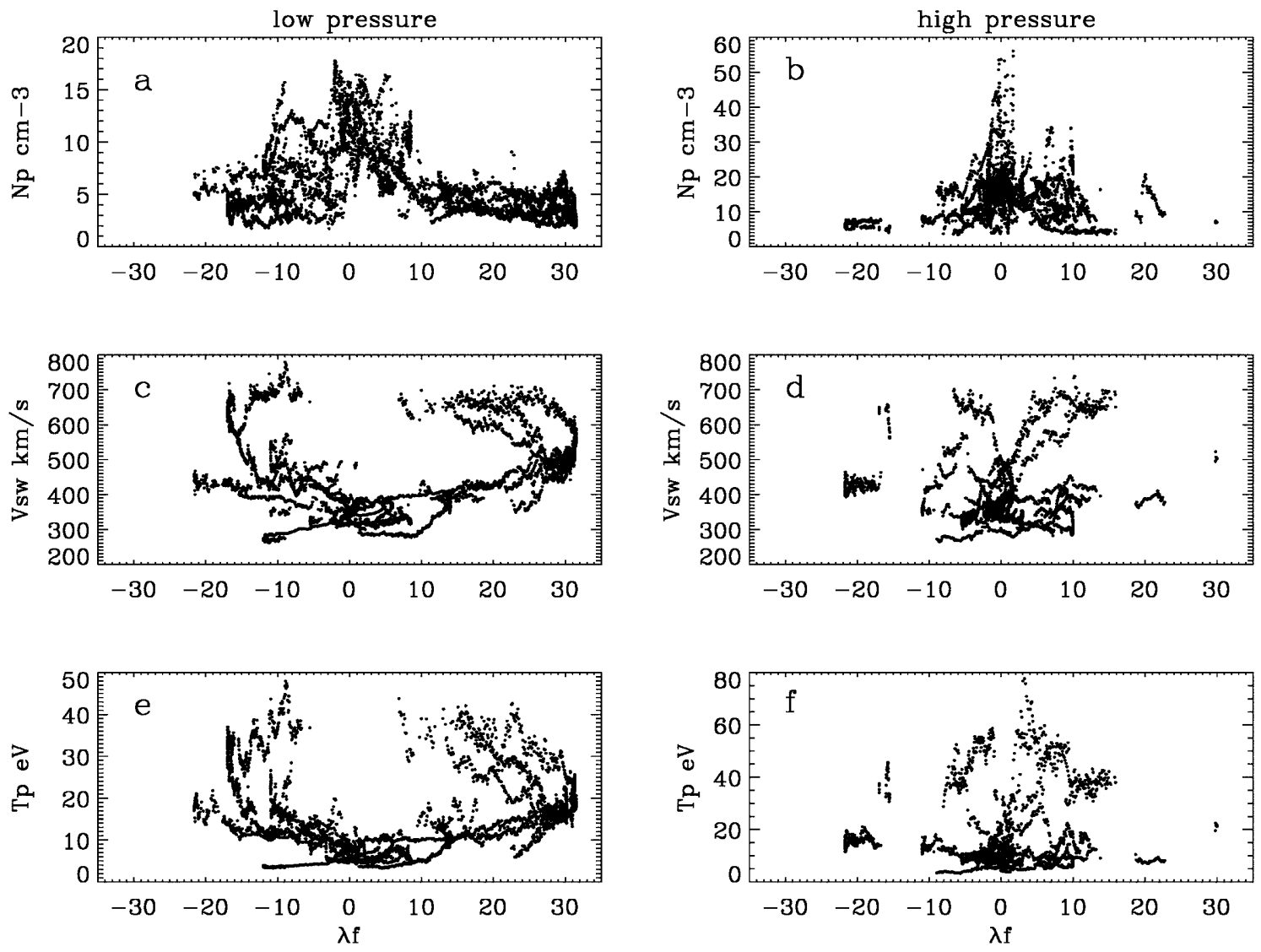

Fig. 5a-f. Seventy consecutive days. Scatter plots of $10 \mathrm{~min}$ averages of $N_{p}, V_{s w}$ and $T_{p}$ in a low-pressure wind (left panels) and in a compressed wind (right panels) as functions of $\lambda_{f}$. The critical pressure

is $P_{T h}+P_{M}=5 \times 10^{-11} \mathrm{~Pa}$. Note that the ordinate scales are not the same in the right and the left panels

line is the average value for a reduced sample (43 days from 135.0 to 178.0) because the fitted latitude $\lambda_{f}$ is probably more precise on these days (Fig. 1a).

The scatter plot and the average of $N_{p}$ as functions of $\lambda_{s e}$ (Fig. 6a, b) are nearly the same before and after the HCS crossing. But $V_{s w}$ and $T_{p}$ as functions of $\lambda_{s e}$ (Fig. 6c, e) are less symmetrical than as functions of $\lambda_{f}$ (Fig. 5c, e). The speed gradient is 5 to $10 \mathrm{~km} / \mathrm{s} /$ degree between $\lambda_{s e}=0^{\circ}$ and $\lambda_{s e}=-30^{\circ}$ or $-20^{\circ}$, and about 15 to $35 \mathrm{~km} / \mathrm{s} /$ degree between $0^{\circ}$ and $10^{\circ}$ or $15^{\circ}$. The asymmetry between the profiles for $\lambda_{s e}<0$ (before the HCS crossing) and $\lambda_{s e}>0$ (after the HCS crossing) reveals that $V_{s w}$ and $T_{p}$ do not only depend on the modulus of the heliomagnetic latitude: the stream interactions, which occur for $\lambda_{s e}>0$ play a role even in the low-pressure wind.

There is a weak minimum of the average of $|B|$ at low latitudes (Fig. 6g, h). The existence of this minimum does not depend on the low pressure condition $P \leq 5 \times 10^{-11} \mathrm{~Pa}$; it is also found (but not shown) for $P \leq 7 \times 10^{-11} \mathrm{~Pa}$.

We note in Fig. 6b that the average density peaks above $10 \mathrm{~cm}^{-3}$ in the heliospheric plasma sheet (HPS) between $\lambda_{\text {se }}=-2.5^{\circ}$ and $2.5^{\circ}$. This peak is embedded in a density halo with $N_{p}>6 \mathrm{~cm}^{-3}$ between $\lambda_{s e}=-10^{\circ}$ and $10^{\circ}$. Such a halo has already been observed by Bavassano et al. (1997) on Helios at 0.9 UA as well as at
0.3 UA. It thus seems to be a permanent feature of the streamer belt, close to a minimum of solar activity. The evidence of the halo is not related to the chosen critical value of the total pressure, $5 \times 10^{-11} \mathrm{~Pa}$; it is still observed for $P \leq 4 \times 10^{-11} \mathrm{~Pa}$. As the peak of $N_{p}$ and the dip of $|B|$ are narrower than the minimum of $T_{p}$ (Fig. $6 \mathrm{~b}, \mathrm{f}, \mathrm{h}$ ) there is a pressure equilibrium between the HPS and the density halo.

\subsection{High-pressure wind}

Scatter plots of the solar wind properties in the highpressure wind are shown in Fig. 7. The values of $V_{s w}$ and $T_{p}$ are not controlled by the latitude, and very large as well as very small values of $V_{s w}$ and $T_{p}$ are found for $\left|\lambda_{s e}\right|<5^{\circ}$. The role played by the stream interactions i.e. the asymmetry before and after the HCS for $V_{s w}$ and $T_{p}$ is larger than in the low-pressure wind. The average value of $V_{s w}$ (Fig. 7d) is minimum for $\lambda_{s e} \simeq-6^{\circ}$, while it was minimum at $\lambda_{s e} \simeq 0^{\circ}$ in the low-pressure wind (Fig. 6d). Thus, the minimum average speed is observed to be close to the HCS in the low-pressure wind, but before the HCS crossing in the high-pressure wind.

The minimum values of the field $|B|<1.5 \gamma$ are found at $\left|\lambda_{\text {se }}\right|<10^{\circ}$, but the maximum values of $|B|$ are also found at low latitudes, $\left|\lambda_{\text {se }}\right|<15^{\circ}$ (Fig. 7g). The $\beta$ factor 

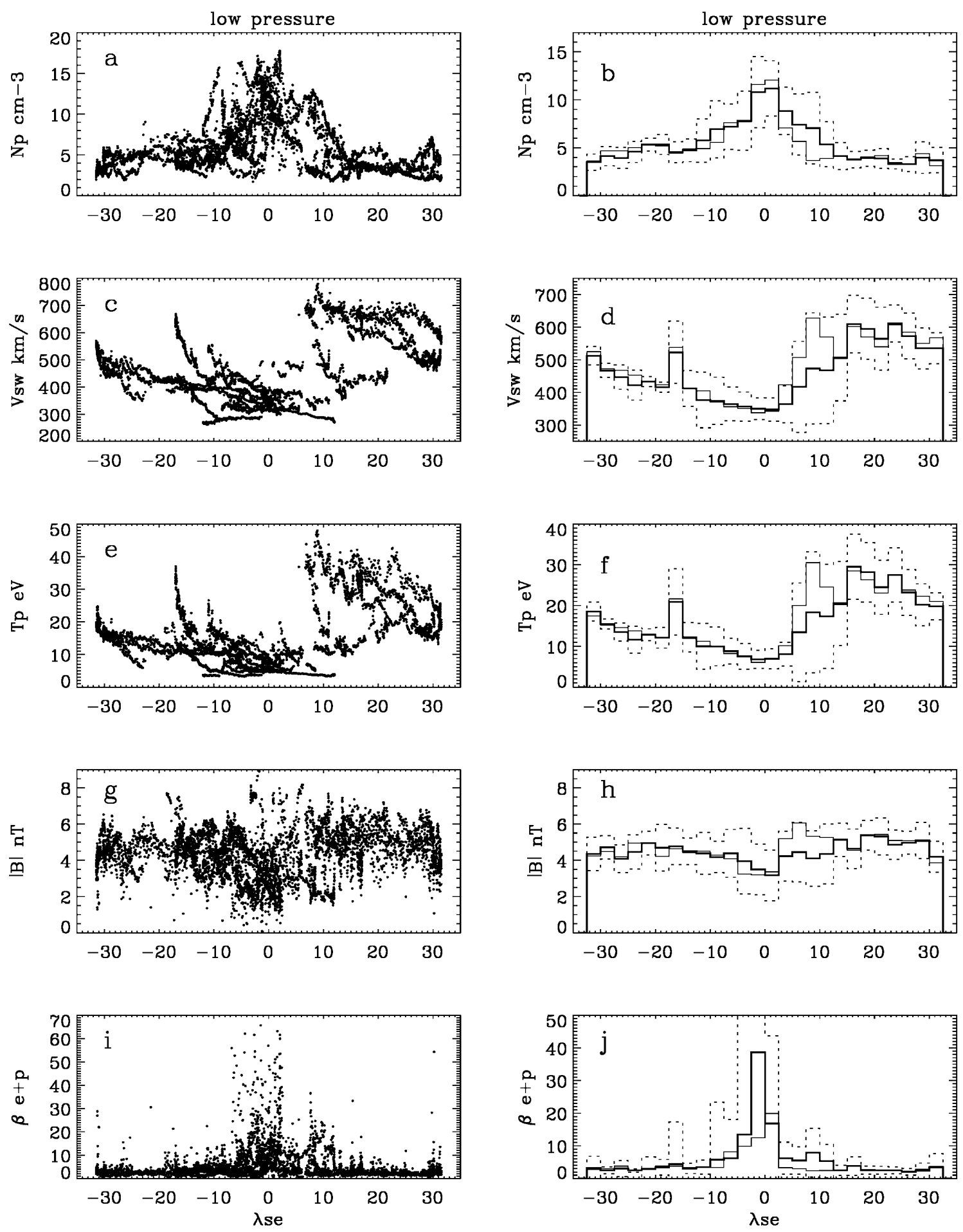

Fig. 6a-j. Seventy consecutive days in the low-pressure solar wind. Left panels: scatter plots of $10 \mathrm{~min}$ averages of the density, the wind speed, the proton temperature, the magnetic field strength and the $\beta$ factor (Eq. 3). The abscissa is the superposed epoch heliomagnetic latitude $\lambda_{\text {se }}$ defined in Sect. 5. Right panels: the thick line and the

$\beta=2 \mu_{0} N_{p} k_{B}\left(T_{e}+T_{p}\right) / B^{2}$

is displayed in Figs. 6 and 7. Both weak and strong values of $\beta$ are found at low $\lambda_{\text {se }}$ in the high-pressure wind (Fig. 7i). But in the low-pressure wind (Fig. 6i) dashed lines show respectively the average of the wind parameters over 70 days, and the average plus or minus the standard deviation for 10 min data, as functions of $\lambda_{\text {se }}$. The thin line is the average over the reduced sample of 43 days (see Sect. 5.1)

there is a general increase of the minimum values of $\beta$, which has a latitudinal thickness of a few degrees. The average value of $\beta$ at low latitude is thus larger in the low-pressure wind (Figs. 6j, 7j). 

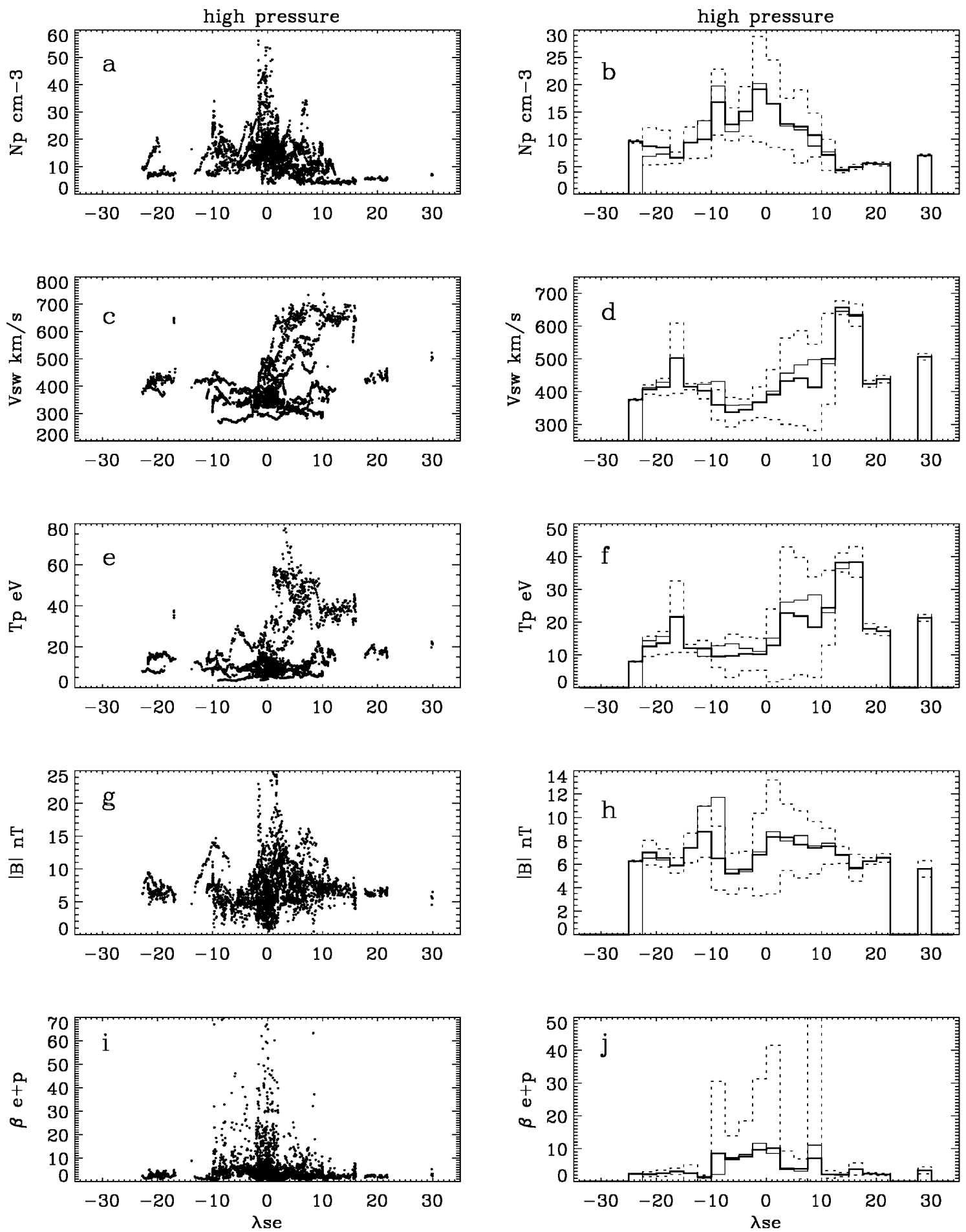

Fig. 7a-j. Seventy consecutive days in the high-pressure solar wind. Left panels: scatter plots of $10 \mathrm{~min}$ averages of the density, the wind speed, the proton temperature, the magnetic field strength, and the $\beta$ factor (Eq. 3). The abscissa is the superposed epoch heliomagnetic latitude $\lambda_{\text {se }}$ defined in Sect. 5. Right panels: the thick line and the

\section{Pressures as functions of the heliomagnetic latitude}

The total pressure $P$ is shown in Fig. 8 as a function of $\lambda_{s e}$ in the low-pressure wind (left panels) and in the highpressure wind (right panels). In these two regimes, $P$ is dashed lines show respectively the average of the wind parameters, and the average plus or minus the standard deviation for $10 \mathrm{~min}$ data, as functions of $\lambda_{\text {se }}$. The thin line is the average over the reduced sample of 43 days

slightly larger $(20 \%)$ after the HCS crossings (Fig. 8c, d). $P$ does not really depend on the latitude in the lowpressure wind (Fig. 8c): at low latitudes, the HPS and its density halo are regions which have the same total pressure as the non-compressed wind at higher latitudes. 
The latitude thickness of the compressed regions $\left(P_{M}+P_{T h}>5 \times 10^{-11} \mathrm{~Pa}\right)$ is about $20^{\circ}$. Thus, the corotating interaction regions do not reach heliomagnetic latitudes larger than about $10^{\circ}$.
In Fig. 9, we display the average values of $P_{M}$ and of $P_{T h}$ in the wind with a low total $P$ (left panels) and a high $P$ (right panels). In the low-pressure wind, there is a small dip of $P_{M}$ and a small bump of $P_{T h}$ in the low-
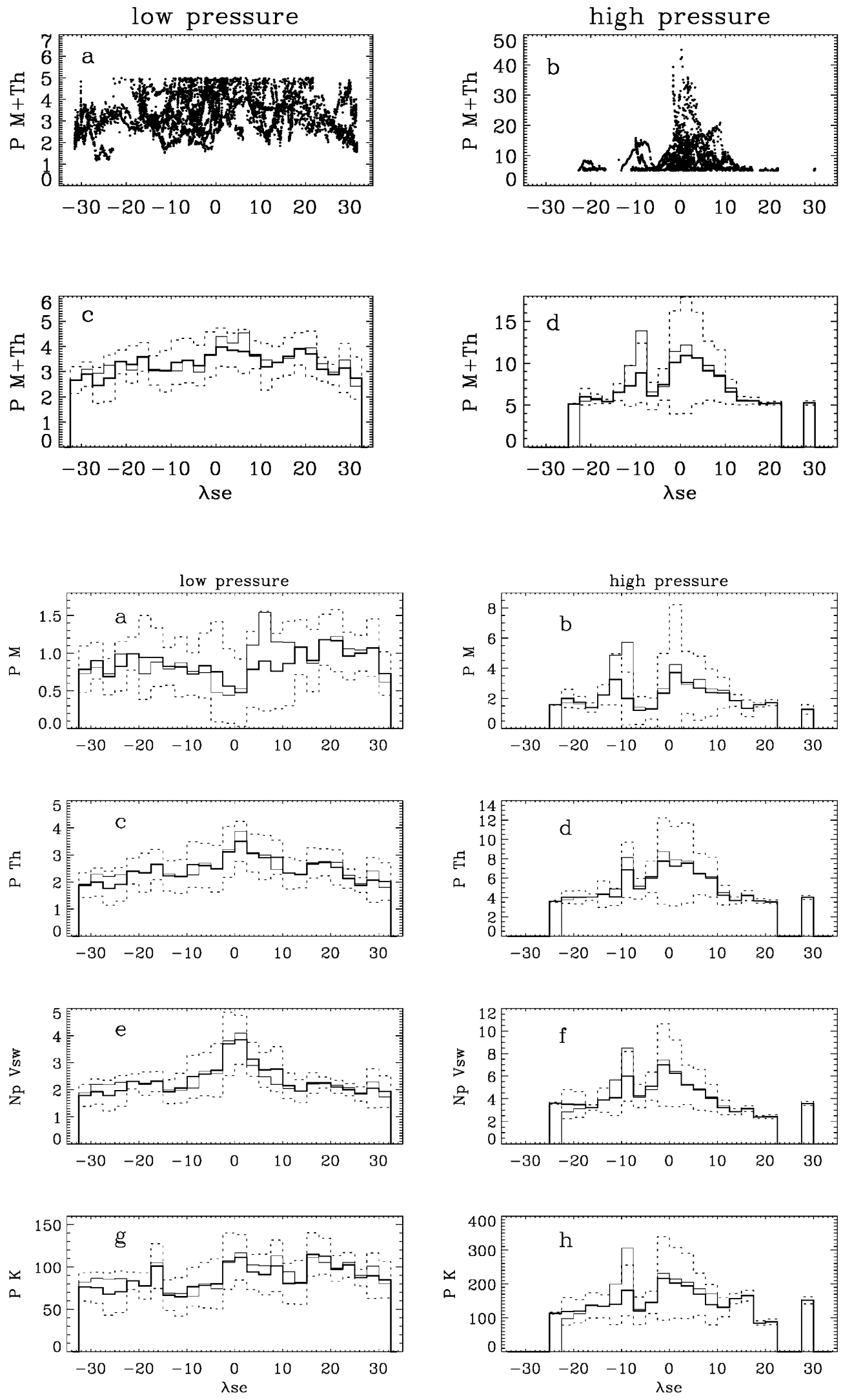
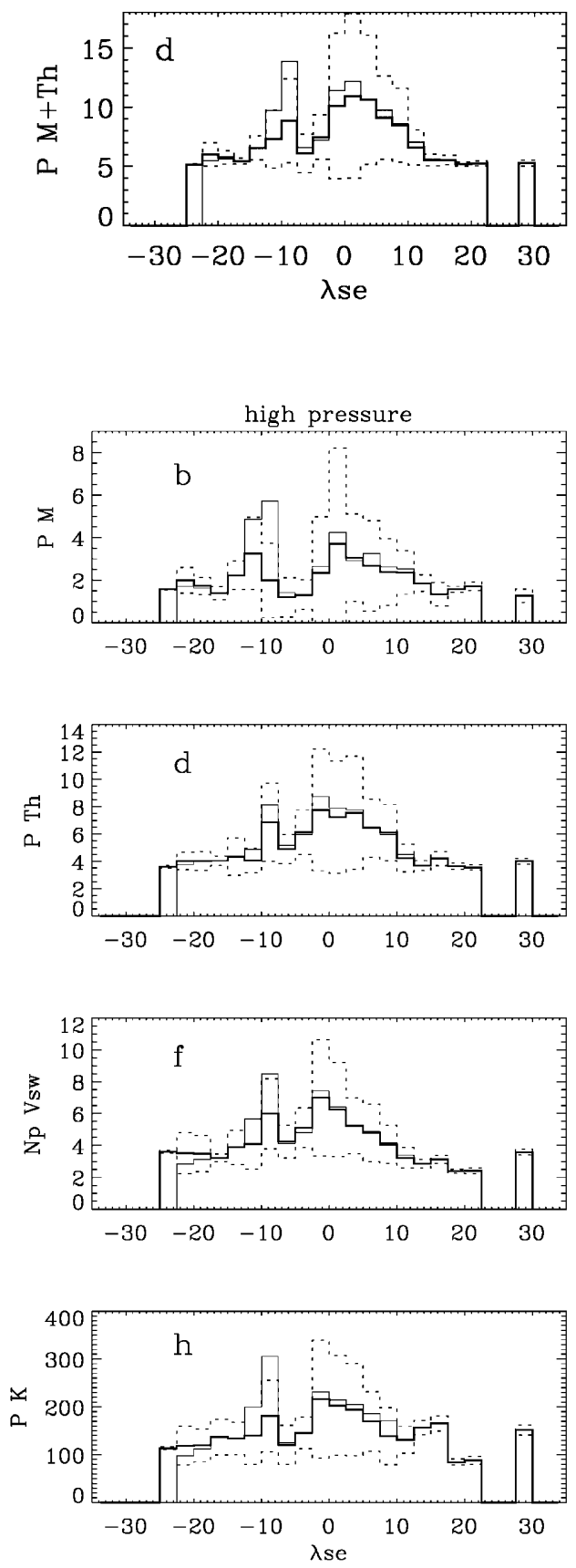

Fig. 8a-d. Seventy consecutive days. Left panels: scatter plot and average value of the total pressure in the low-pressure wind $P \leq 5 \times 10^{-11} \mathrm{~Pa}$. Right panels: the same quantities in the high pressure wind. The thick line and the dashed lines are respectively the average of the wind parameters, and the average plus or minus the standard deviation for $10 \mathrm{~min}$ data. The thin line is the average over 43 days. The pressure unit is $10^{-11} \mathrm{~Pa}$
Fig. 9a-h. The thick line and the dashed lines are respectively the average of the wind parameters over 70 days, and the average plus or minus the standard deviation for $10 \mathrm{~min}$ data. The thin line is the average over 43 days. From top to bottom: the magnetic and the thermal pressures, the particle flux in $10^{6} \mathrm{~m}^{-2} \mathrm{~s}^{-1}$, and the kinetic pressure. Left panels: in the low-pressure wind. Right panels: in the high-pressure wind. The pressure unit is $10^{-11} \mathrm{~Pa}$. Note that the ordinate scales are not the same in the right and the left panels 
latitude plasma sheet. In the high-pressure wind, the latitudinal thickness of the bump of $P_{T h}$ is less than $20^{\circ}$ (Fig. 9d), while it was more than $90^{\circ}$ for $P_{T h}$ measured on Ulysses as a function of the heliographic latitude and averaged over a solar rotation (Suess et al., 1996).

The particle flux $N_{p} V_{s w}$ is shown in Fig. 9e, f. The flux peaks at low latitude in the low-pressure wind, and even more in the high-pressure wind. The kinetic pressure (Fig. 9g, h)

$P_{K}=1 / 2 N_{p} m_{p} V_{s w}^{2}$

does not really depend on the latitude (Bruno et al., 1986). It tends to be weaker before the HCS crossing, and larger after.

We conclude that in the low-pressure solar wind $\left(P_{T h}+P_{M} \leq 5 \times 10^{-11} \mathrm{~Pa}\right)$ i.e. outside of the stream interaction regions, there are two main kinds of plasma: a heliospheric plasma sheet in which $N_{p}$ is high, and $T_{p}$, $V_{s w}$ and $|B|$ are low, and the fast wind which is hotter and less dense, with a slightly larger $|B|$. However, even in the low-pressure wind, the asymmetry of the latitudinal profiles of $V_{s w}$ and $T_{p}$ before and after the HCS crossing indicates the part played by the stream interactions from the Sun to $1 \mathrm{AU}$ at heliomagnetic latitudes $|\lambda| \leq 30^{\circ}$. The high-pressure wind is mainly confined to $|\lambda| \leq 10^{\circ}$. A comparison of the left panels of Fig. 7 with the left panels of Fig. 6 shows that the latitudinal dependence of the parameters is less clear in the high-pressure wind.

\section{Discussion}

The interval considered ( 70 days) is relatively short. But it is the longest continuous interval without magnetic clouds or strong shocks sampled by Wind in the solar wind over two years. Our fitting of the latitude of Wind with respect to the HCS relies on the estimations of the classic model (Fig. 1a). According to Neugebauer et al. (1998) these estimations agree relatively well with the HCS crossings observed both on Ulysses and on Wind in early 1995 (see also Crooker et al., 1997). A good overall agreement between the predictions of the classic model and the sector boundary crossings by Wind has also been noted by Lepping et al. (1996).

Erdös and Balogh (1998), analysing the Ulysses data from February to April 1995, compare the observed HCS crossings to two models of the solar neutral line: the classic model used here, and the radial model (in which the magnetic field is assumed to be radial above the photosphere). The radial model only predicts three of the seven observed HCS crossings: it fails to predict the four crossings in the Northern Hemisphere because it is not warped enough. The classic model is better: it predicts five of the seven HCS crossings in both hemispheres. But it is too warped in the Southern Hemisphere: while the latitude of Wind with respect to the HCS reaches $\lambda_{H} \simeq 30^{\circ}$ with the classic model (Fig. 1a), the radial model would only give $20^{\circ}$. Had we used the radial model, the latitudinal scale of our figures and the latitudinal thickness of the HPS would have been divided by a factor 1.5.
The overall direction of the total electron heat flux has been used to identify the sector boundary crossings. McComas et al. (1989), using the heat flux of only the electron halo (ISEE 3 data) found a close association between heat flux dropouts (below $2 \mu \mathrm{W} / \mathrm{m}^{2}$ ) and sector boundaries. As shown in Fig. 1d, the total heat flux does not drop at the sector boundary or HCS crossings. Also using the halo heat flux on ISEE 3, Kahler et al. (1998) found that $a z_{B}-a z_{Q}$ was always close to $180^{\circ}$ or $360^{\circ}$ while our Fig. 1c displays a total heat flux less clearly aligned with the magnetic field. These differences may be due to the fact that we have analysed the total heat flux, not the halo heat flux. They also may be due to uncertainties in the calculation of the direction and modulus of the total electron heat flux (C. Salem et al., 2000, to be submitted to J. Geophys. Res.). The solar heat flux can also be perturbed by electrons backstreaming sunward when the magnetic field line through the spacecraft intersects the Earth's bow shock (Stansberry et al., 1988): according to Berdichevsky et al. (1999), Wind was in the proton foreshock during at least six periods (lasting 1 to $2 \mathrm{~h}$ ) of our interval. Szabo et al. (1999) analysed the azimuth of the magnetic field and the heat flux of the electron halo $(E>90 \mathrm{eV})$ during two long periods of the Wind data, including the period of the present study. They found, as we did, that only some of the current sheet crossings were true HCS crossings, with a heat flux which remains antisunward. However, as explained in Sect. 3, we have not analysed the HCS on a small scale; we have assumed that all the current sheets observed between two sectors with opposite magnetic polarities and an antisunward heat flux were at low heliomagnetic latitude.

In the present study, we have considered the heliomagnetic latitude $\lambda$ of the observer and not the angular distance $\delta$ perpendicular to the HCS. According to Wang et al. (1997) the solar wind speed observed on Ulysses depends on $\lambda$ (through the magnetic flux tube expansion factor) more than on $\delta$.

We have found a size of at least $5^{\circ}$ in latitude for the overdensity in the low pressure (non-compressive) plasma close to the HCS. The corresponding scale is $13 \times 10^{6} \mathrm{~km}(\simeq 0.1 \mathrm{AU})$ at $1 \mathrm{AU}$. Winterhalter et al. (1994) estimated the thickness of the regions where the $\beta$ factor is significantly enhanced close to the HCS, at the Lagrange point; they probably considered all the regions with an enhanced $\beta$, including the compressed regions close to the HCS. This thickness does not rely on a HCS model because it was estimated with the local normal to the HCS, the wind velocity along the normal and the event duration. Winterhalter et al. (1994) found a thickness of 200 to $400 \times 10^{3} \mathrm{~km}$, i.e. 30 to 100 times smaller than the present size. Our Fig. 6i shows that there are small scale peaks of $\beta$ superimposed on a broader peak where the minimum value of $\beta$ increases; these two latitudinal scales correspond to the two very different thicknesses found by Winterhalter et al. (1994) and in the present work (see also Crooker, 1999; Shodhan et al., 1999). However, a reason for the discrepancy between the results of Winterhalter et al. (1994) and ours may also be the large uncertainty on $\lambda$, 
which is at least $5^{\circ}$. The latitudinal thickness shown in Fig. $4 \mathrm{~b}$ is probably an upper limit. It can be artificially increased by the uncertainty on $\lambda$. Bavassano et al. (1997) have considered the longitudinal thickness of the HPS: this thickness is about $2^{\circ}$, and the thickness of the density halo is about $15^{\circ}$ to $20^{\circ}$. These observations are more consistent with ours, and they do not rely on a model of the HCS.

Several models have been proposed for the variations of the solar wind properties with the heliographic latitude $\lambda_{G}$. These models are steady and axisymmetric, with a larger density and a weaker speed at low $\lambda_{G}$, i.e. with a flat heliospheric plasma sheet at small $\left|\lambda_{G}\right|$ (Lima and Tsinganos, 1996; Suess et al., 1996). These models assume that the thermal pressure bump, the overdensity and the minimum speed at low $\lambda_{G}$ exist continuously from the Sun to $1 \mathrm{AU}$, with an axisymmetric self-similar shape for the wind properties averaged over a solar rotation. They neglect the effect of the stream interactions. We have shown in Sect. 5 that even in the lowpressure wind there are indications of the effect of the stream interactions. It is thus not relevant to compare our Wind data to axisymmetric latitudinal solar wind models.

A more relevant model for our data is the model of Pizzo (1994) for the deformation of the heliospheric current sheet in a 3D MHD numerical model of the solar wind. In this model, a solar wind structure with a tilted dipole is imposed close to the Sun, with a flat HCS. In heliographic coordinates, this flat HCS appears as a two-sector sinusoidal HCS. At high latitudes, the wind is fast, hot and tenuous; a narrow band of slow, cold and dense flow (the HPS) surrounds the HCS. Large-scale dynamical interactions produce a deformation of the HCS and of the HPS. Pizzo (1994) does not show the results of the model at $1 \mathrm{AU}$; the speed pattern at $1 \mathrm{AU}$ is shown by Pizzo and Gosling (1994). At 5 AU (Fig. 1 in Pizzo, 1994) the speed and the density profiles, as functions of the latitude with respect to the HCS, are not the same at the heliographic longitudes of the compressed regions and at the heliographic longitudes of regions which are less compressed.

An interesting indication in the model of Pizzo (1994) is that, at least at $5 \mathrm{AU}$, the speed minimum does not occur at the HCS crossing, except for a narrow range of heliographic longitudes. As shown in Sect. 5, the minimum average speed is observed to be close to the HCS in the low-pressure wind, but before the HCS crossing in the high-pressure wind. Thus, the model of Pizzo (1994) qualitatively matches our Wind observations, even if the differences between the compressed and the non compressed regions, in the model at $1 \mathrm{AU}$, are much weaker than the observed differences.

The structure of the overdense regions has a strong influence on the propagation of radio waves whose frequency is a few tens of $\mathrm{kHz}$, just above the electron plasma frequency. For instance, a terrestrial kilometric radiation is sometimes observed from the Lagrange point as an isotropic radiation. This isotropisation depends on the density fluctuations. Studies of the density fluctuations have been carried out in different solar wind regions (Bavassano et al., 1997) or with the criteria of the wind speed and of the $\beta$ factor (Huddleston et al., 1995). The two criteria of the wind speed and of the total pressure seem to be better to characterise the density fluctuations: we shall present, in a forthcoming paper, a study of the density fluctuations observed on Wind. The isotropisation of the radiation implies not only the usual scattering of radio waves in the interplanetary medium, but sometimes the presence of strongly refracting/scattering density structures (Steinberg et al., 1990, 2000). To study quantitatively the propagation of these low frequency radio waves, it is important to know whether the overdensities are discontinuous or form a continous wall for radio waves at low latitudes. The high-density regions probed by interplanetary scintillations are not continuous (Jackson et al., 1998). We have found that there were more or less strong overdensities at each HCS crossing, so that the heliospheric plasma sheet is a continuous density wall but with a variable height. According to Pizzo (1994), the low-pressure overdensity which surrounds the HCS remains close to the HCS from the Sun to $5 \mathrm{AU}$ at low heliographic latitudes; but the greatest concentrations of plasma at $5 \mathrm{AU}$ are found at high heliographic latitudes, further from the equator than the HCS itself.

\section{Conclusion}

We have considered the distribution of several solar wind parameters as functions of the fitted latitude $\lambda_{f}$ of Wind with respect to the heliospheric current sheet (HCS). The hypothetical position of the HCS has been deduced from the classic model of Hoeksema et al. $(1982,1983)$ and improved by the Wind observations of the current sheet crossings and of the direction of the total electron heat flux. We have found that the variations with $\lambda_{f}$ of several wind parameters are not the same in two kinds of wind: the low pressure wind where the total pressure $P$ (the thermal plus magnetic pressure) is lower than about $5 \times 10^{-11} \mathrm{~Pa}$, and the compressed wind where $P>5 \times 10^{-11} \mathrm{~Pa}$. The compressed regions are mainly observed for $\left|\lambda_{f}\right|$ lower than about $10^{\circ}$ : the corotating interaction regions do not reach higher heliomagnetic latitudes. In this highpressure wind, there is a strong scatter of the wind parameters at a given $\lambda_{f}$. On the contrary, in the lowpressure wind, the plasma properties are much tighter functions of the heliomagnetic latitude: at low values of $\left|\lambda_{f}\right|$, close to the HCS, there is a continuous overdense sheet about $5^{\circ}$ thick, with a density of 10 to $20 \mathrm{~cm}^{-3}$; this sheet is embedded in a halo whose density is 5 to $10 \mathrm{~cm}^{-3}$ and latitudinal thickness about $20^{\circ}$. At larger values of $\left|\lambda_{f}\right|$, the density drops to 3 to $5 \mathrm{~cm}^{-3}$, close to the solar wind density at high heliographic latitudes. In the overdense sheet and the halo, $V_{s w}$ and $T_{p}$ are smaller than at higher latitudes and the average $\beta$ factor is larger; in the overdense sheet, the thermal pressure is slightly higher and the magnetic pressure slightly lower. Outside the compressed regions, there is thus a pressure equilibrium between the high-latitude dilute wind, a 
low-latitude overdense sheet and its halo. Our results illustrate the fact that the total pressure is better than the $\beta$ factor to characterise the solar wind regimes.

Close to the Sun, the solar wind properties are function of a single parameter, the proton temperature or the wind speed, depending on the heliomagnetic latitude $\lambda$. At $1 \mathrm{AU}$, because of the stream interactions, the wind properties are functions of two parameters which are $\lambda$ and the total pressure. This can be understood on the basis of the work of Pizzo (1994) who developed a solar wind numerical model where, at a given distance from the Sun, the properties of the tilted heliospheric plasma sheet are functions of two parameters, the heliographic longitude and $\lambda$. Our findings at $1 \mathrm{AU}$ are qualitatively compatible with this model: the observed properties of the heliospheric plasma sheet depend on $\lambda$; but they also depend on the heliographic longitude which is itself related to the pressure, high in the stream interaction regions, and low elsewhere.

Whatever the solar wind pressure, there is an asymmetry of the latitudinal profiles of $V_{s w}$ and $T_{p}$ before and after the HCS crossing. In the low-pressure wind, the latitudinal gradient of the wind speed is 5 to $10 \mathrm{~km} / \mathrm{s} /$ degree before the HCS crossing, and about 15 to $35 \mathrm{~km} / \mathrm{s} /$ degree after the HCS crossing. This asymmetry is an effect of the stream interaction, which is thus observable even in the low-pressure wind.

Finally, let us recall that we have considered 2.5 solar rotations in May to July 1995, close to the minimum of the solar cycle. The latitudinal distribution of the solar wind parameters can change strongly along the solar cycle.

Acknowledgements. We are very grateful to the teams of the Solar Wind experiment (PI K.W. Ogilvie), of the Magnetic Field Investigation experiment (PI R.P. Lepping) and of the Three Dimensional Plasma experiment (PI R.P. Lin). We thank T.R. Sanderson for the poster A summary of interplanetary conditions observed by WIND in 1995, and J.T. Hoeksema for the computations of the position of the Sun's neutral line. We thank the referees for very useful comments and questions. The French contribution is supported by the Centre National d'Etudes Spatiales and the Centre National de la Recherche Scientifique.

Topical Editor E. Antonucci thanks B. Bavassano and M. Neugebauer for their help in evaluating this paper.

\section{References}

Bavassano, B., R. Woo, and R. Bruno, Heliospheric plasma sheet and coronal streamers, Geophys. Res. Lett., 24, 1655-1658, 1997.

Berdichevsky, D., G. Thejappa, F. R. Fitzenreiter, R. L. Lepping, T. Yamamoto, S. Kokobun, R. W. McEntire, D. J. Williams, and R. P. Lin, Widely spaced wave-particle observations during GEOTAIL and Wind magnetic conjunctions in the Earth's ion foreshock with near-radial interplanetary magnetic field, J. Geophys. Res., 104, 463-482, 1999.

Borrini, G., J. T. Gosling, S. J. Bame, W. C. Feldman, and J. M. Wilcox, Solar wind helium and hydrogen structure near the heliospheric current sheet: a signal of coronal streamers at 1 AU, J. Geophys. Res., 86, 4565-4573, 1981.

Bruno, R., U. Villante, B. Bavassano, R. Schwenn, and F. Mariani, In-situ observations of the latitudinal gradients of the solar wind parameters during 1976 and 1977, Solar Phys., 104, 431445, 1986.

Crooker, N. U., Heliospheric current sheet structure, Solar Wind Nine, Eds. S.R. Habbal et al., AIP, 93-98, 1999.

Crooker, N. U., G. L. Siscoe, S. Shodhan, D. F. Webb, J. T. Gosling, and E. J. Smith, Multiple heliospheric current sheets and coronal streamer belt dynamics, J. Geophys. Res., 98, 93719381, 1993.

Crooker, N. U., M. E. Burton, G. L. Siscoe, S. W. Kahler, J. T. Gosling, and E. J. Smith, Solar wind streamer belt structure, J. Geophys. Res., 101, 24 331-24 341, 1996.

Crooker, N. U., A. J. Lazarus, J. L. Phillips, J. T. Steinberg, A. Szabo, R. P. Lepping, and E. J. Smith, Coronal streamer belt asymmetries and seasonal solar wind variations deduced from Wind and Ulysses data, J. Geophys. Res., 102, 4673-4679, 1997.

Erdös, G., and A. Balogh, The symmetry of the heliospheric current sheet as observed by Ulysses during the fast latitude scan, Geophys. Res. Lett., 25, 245-248, 1998.

Feldman, W. C., J. R. Asbridge, S. J. Bame, M. D. Montgomery, and S. P. Gary, Solar wind electrons, J. Geophys. Res., 80, 41814196, 1975.

Gosling, J. T., E. Hildner, J. R. Asbridge, S. J. Bame, and W. C. Feldman, Noncompressive density enhancements in the solar wind, J. Geophys. Res., 82, 5005-5010, 1977.

Gosling, J. T., G. Borrini, J. R. Asbridge, S. J. Bame, W. C. Feldman, and R. T. Hansen, Coronal streamers in the solar wind at 1 AU, J. Geophys. Res., 86, 5438-5448, 1981.

Hoeksema, J. T., J. M. Wilcox, and P. H. Scherrer, Structure of the heliospheric current sheet in the early portion of sunspot cycle 21, J. Geophys. Res., 87, 10 331-10 338, 1982.

Hoeksema, J. T., J. M. Wilcox, and P. H. Scherrer, The structure of the heliospheric current sheet: 1978-1982, J. Geophys. Res., 88, 9910-9918, 1983.

Huddleston, D. E., R. Woo, and M. Neugebauer, Solar wind density structure at $1 \mathrm{AU}$ and comparison to Doppler scintillation measurements, Solar Wind Eight, Eds. D. Winterhalter et al., AIP, 530-533, 1996.

Hundhausen, A. J., S. J. Bame, J. R. Asbridge, and S. J. Sydoriak, Solar wind proton properties: Vela observations from July 1965 to June 1967, J. Geophys. Res., 75, 4643-4657, 1970.

Issautier, K., N. Meyer-Vernet, M. Moncuquet, and S. Hoang, Poleto-pole solar wind density from Ulysses radio measurements, Solar Phys., 172, 335-343, 1997.

Jackson, B. V., P. L. Hick, M. Kojima, and A. Yokobe, Heliospheric tomography using interplanetary scintillation observations 1. Combined Nagoya and Cambridge data, J. Geophys. Res., 103, 12 049-12 067, 1998.

Kahler, S., N. U. Crooker, and J. T. Gosling, Properties of interplanetary sector boundaries based on electron heat-flux flow directions, J. Geophys. Res., 103, 20 603-20 612, 1998.

Lepping, R. P., M. H. Acna, L. F. Burlaga, W. M. Farrell, J. A. Slavin, K. H. Schatten, F. Mariani, N. F. Ness, F. M. Neubauer, Y. C. Whang, J. B. Byrnes, R. S. Kennon, P. V. Panetta, J. Scheifele, and E. M. Worley, The WIND magnetic field investigation, Space Sci. Rev., 71, 207-229, 1995.

Lepping, R. P., A. Szabo, M. Peredo, J. T. Hoeksema, Large-scale properties and solar connection of the heliospheric current and plasma sheets: WIND observations, Geophys. Res. Lett., 23, 1199-1202, 1996.

Lima, J., and K. Tsinganos, The heliolatitudinal gradient of the solar wind during solar minimum conditions modelled by exact hydrodynamic solutions, Geophys. Res. Lett., 23, 117-120, 1996.

Lin, R. P., K. A. Anderson, S. Ashford, C. Carlson, D. Curtis, R. Ergun, D. Larson, J. McFadden, M. McCarthy, G. K. Parks, H. Rème, J.-M. Bosqued, J. Coutelier, F. Cotin, C. d'Uston, K.-P. Wenzel, T. R. Sanderson, J. Henrion, J. C. Ronnet, G. Paschmann, A three-dimensional plasma and energetic particle investigation for the WIND spacecraft, Space Sci. Rev., 71, 125-153, 1995. 
Ma, G., K. Marubashi, and T. Maruyama, Heliospheric current sheet inclinations at Venus and Earth, Ann. Geophysicae, 17, 642-649, 1999.

McComas, D. J., J. T. Gosling, J. L. Phillips, S. J. Bame, J. G. Luhmann, and E. J. Smith, Electron heat flux dropouts in the solar wind: evidence for interplanetary magnetic field reconnection?, J. Geophys. Res., 94, 6907-6916, 1989.

Neugebauer, M., R. J. Forsyth, A. B. Galvin, K. L. Harvey, J. T. Hoeksema, A. J. Lazarus, R. P. Lepping, J. A. Linker, Z. Mikic, J. T. Steinberg, R. von Steiger, Y.-M. Wang, and R. F. WimmerSchweingruber, Spatial structure of the solar wind and comparisons with solar data and models, J. Geophys. Res., 103, 14 58714 599, 1998.

Ogilvie, K. W., D. J. Chornay, R. J. Fitzenreiter, F. Hunsaker, J. Keller, J. Lobell, G. Miller, J. D. Scudder, E. C. Sittler, R. B. Torbert, D. Bodet, G. Needell, A. J. Lazarus, J. T. Steinberg, J. H. Tappan, A. Mavretic, and E. Gergin, SWE, a comprehensive plasma instrument for the WIND spacecraft, Space Sci. Rev., 71, 55-77, 1995.

Phillips, J. L., S. J. Bame, A. Barnes, B. L. Barraclough, W. C. Feldman, B. E. Goldstein, J. T. Gosling, G. W. Hoogeveen, D. J. McComas, M. Neugebauer, and S. T. Suess, Ulysses solar wind plasma observations from pole to pole, Geophys. Res. Lett., 22, 3301-3304, 1995.

Pizzo, V. J., Global quasi-steady dynamics of the distant solar wind 2. Deformation of the heliospheric current sheet, J. Geophys. Res., 99, 4185-4191, 1994.

Pizzo, V. J., and J. T. Gosling, 3-D simulation of high-latitude interaction regions: comparison with Ulysses results, Geophys. Res. Lett., 21, 2063-2066, 1994.

Sanderson, T. R., R. P. Lin, D. E. Larson, M. P. McCarthy, G. K. Parks, J.-M. Bosqued, N. Lormant, K. W. Ogilvie, R. P. Lepping, A. Szabo, A. J. Lazarus, J. T. Steinberg, and J. T. Hoeksema, WIND observations of the influence of the
Sun's magnetic field on the interplanetary medium at 1 AU, J. Geophys. Res., 103, 17 235-17 247, 1998.

Scime, E. E., S. J. Bame, W. C. Feldman, S. P. Gary, J. L. Phillips, and A. Balogh, Regulation of the solar wind electron heat flux from 1 to 5 AU: Ulysses observations, J. Geophys. Res., 99, 23 401-23 410, 1994.

Shodhan, S., N. U. Crooker, R. J. Fitzenreiter, R. P. Lepping, and J. T. Steinberg, Density enhancements in the solar wind, Solar Wind Nine, Eds. S.R. Habbal et al., AIP, 601-604, 1999.

Stansberry, J. A., J. T. Gosling, M. F. Thomsen, S. J. Bame, and E. J. Smith, Interplanetary magnetic field orientations associated with bidirectional electron heat fluxes detected at ISEE 3, J. Geophys. Res., 93, 1975-1980, 1988.

Steinberg, J.-L., S. Hoang, and J.-M. Bosqued, Isotropic terrestrial kilometric radiation: a new component of the Earth's radio emission, Ann. Geophysicae, 8, 671-686, 1990.

Steinberg, J.-L., C. Lacombe, and S. Hoang, Isotropization of the terrestrial low frequency radio bursts, (in press) J. Geophys. Res., 2000.

Suess, S. T., E. J. Smith, J. L. Phillips, B. E. Goldstein, and S. Nerney, Latitudinal dependence of the radial IMF component - interplanetary imprint, Astron. Astrophys., 316, 304-312, 1996.

Szabo, A., D. E. Larson, and R. P. Lepping, The heliospheric current sheet on small scale, Solar Wind Nine, Eds. S.R. Habbal et al., AIP, 589-592, 1999.

Wang, Y.-M., N. R. Shelley, J. L. Phillips, and B. E. Goldstein, Solar wind stream interactions and the wind speed-expansion factor relationship, Astrophys. J., 488, L51-L54, 1997.

Winterhalter, D., E. J. Smith, M. E. Burton, N. Murphy, and D. J. McComas, The heliospheric plasma sheet, J. Geophys. Res., 99, 6667-6680, 1994.

Zhao, X.-P. and A. J. Hundhausen, Organization of solar wind properties in a tilted, heliomagnetic coordinate system, J. Geophys. Res., 86, 5423-5430, 1981. 